\title{
LX. On the nebular hypothesis.-II. Interaction
}

\section{Pliny Earle Chase}

To cite this article: Pliny Earle Chase (1876) LX. On the nebular hypothesis. - II. Interaction , Philosophical Magazine Series 5, 1:7, 507-510, DOI: 10.1080/14786447608639075

To link to this article: http://dx.doi.org/10.1080/14786447608639075

$$
\text { 曲 Published online: } 13 \text { May } 2009 .
$$

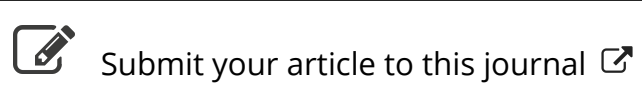

\footnotetext{
Џ Article views: 2
}

Q View related articles $₫$ 
all star-masses at a stage of their development exhibit a phase of volcanic activity. And it is possible that during this period many of those masses which are of comparatively trifling magnitude may have been partially or wholly broken up and resolved into small fragments.

LX. On the Nebular Hypothesis.-II. Interaction. By PLINY Earle Chase, Professor of Philosophy in Haverford College.

[Continued from p. 319.]

TTHE tendency of vibrations in elastic media to produce har1 monic vibrations, combined with the tendency to geometrical and harmonic as well as to arithmetical progressions, should prepare us to look for relations of various complexity when we study the mutual actions and reactions of planetary masses.

The triangular numbers, $1,1+2,1+2+3,1+2+3+4$, appear as indices in the following equation among powers of the masses of the five principal bodies in the solar system :-

$$
\left(\frac{\Psi}{\odot}\right)^{x} \times\left(\frac{\odot}{\odot}\right)^{3} \times\left(\frac{4}{\odot}\right)^{6}=\left(\frac{h}{\odot}\right)^{10} \text {. }
$$

This appears to be the first equation ever discovered which introduces the products of powers of masses in so systematic a form *. Although its full interpretation may be at present beyond our reach, we can catch glimpses of its meaning, and we may feel a reasonable assurance that it represents some important functional law of equilibrating tendencies between centripetal and centrifugal forces. The truth of the equation is, of course, independent of any assumption with regard to the proper unit of comparison; but the dominance of the solar influence lends interest to the æsthetic harmony afforded by its introduction. This interest is increased by the accordance between the order of position and the magnitude of the indices in the left-hand member of the equation, and by the fact that the nebular centre of planetary inertia $\left(\sqrt{\Sigma m r^{2}} \div \bar{\Sigma} m r\right)$ is in Saturn's orbit.

There is still some uncertainty as to the masses of Neptune and Uranus; so that it is impossible to tell how close this agreement may be; but the deviation from precise accuracy cannot be large. According to Newcomb's latest determinations of

* Laplace, however (Méc. Cél. II. vol. viii. pp. 65-69; VI. vol. ii. pp. $12-16, \& c$.$) , investigated inequalities depending on squares and products$ of the disturbing forces. In his discussions of the Jovian and Saturnian systems he introduced terms containing the third and fifth dimensions of eccentricities and inclinations. 
those masses*, from Neptune's satellite and from perturbations of Uranus, the equation gives two values for Saturn's mass, one of which is slightly larger, the other slightly smaller than Bessel's value. There are, however, other relations of a similar character which can be measured with great accuracy.

Laplace found that if the mass of each planet be multiplied by the product of the square of the eccentricity and the square root of the mean distance, the sum of all the products will always retain the same magnitude; also that if each of the masses be multiplied by the product of the square of the orbital inclination and the square root of the mean distance, the sum of the products will always remain invariable. Now the square root of the mean distance varies inversely as the velocity of circular revolution at the mean distance, or inversely as the square root of the velocity of nucleal rotation at the same distance. It is therefore probable that the primitive nucleal undulations, to which I have hypothetically attributed both the relative positions and the relative masses of the planetary orbs, may have left their record in many other directions than those which I have already pointed out.

Stockwell has found $\dagger$ the following relations :-

"I. The mean motion of Jupiter's perihelion is exactly equal to the mean motion of the perihelion of Uranus; and the mean longitudes of those perihelia differ by exactly $180^{\circ}$.

"II. The mean motion of 'Jupiter's node on the invariable plane is exactly equal to that of Saturn; and the mean longitudes of these nodes differ by exactly $180^{\circ}$."

If the four great planets were ranged in a line, Jupiter on one side of the sun and the remoter planets on the other, the tidal influences, relatively to the nucleal centre of inertia, would drive Jupiter, Uranus, and Neptune to their respective aphelia. Those positions would accord with Stockwell's two theorems, they would approximate the planetary centre of inertia very closely to Saturn's mean radius vector, and they would make the above equation of the products of triangular powers applicable to vector radii as well as to masses; for the logarithms of mean vector radii and of their designated triangular powers, according to Stockwell $\ddagger$, are :-

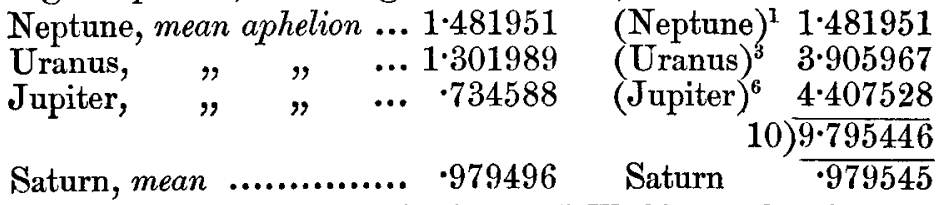

* "The Uranian and Neptunian Systems," Washington Obs. for 1873, App. I.

$\uparrow$ Smithsonian Contributions, 232, p. xiv.

$\ddagger$ Ibid. p. 5 . 
The difference between the actual value of $\log r r . v e c$. and the value as found by the equation $\Psi^{1} \times 0^{3} \times 4^{6}=h^{10}$, is therefore only $\cdot 000049$, representing a numerical difference of only $\frac{1}{80}$ of 1 per cent.

When the hypothetical nebular condensation had proceeded so far as to show the controlling planetary influence of Jupiter's mass, the mean perihelia of Saturn and Uranus were so fixed as to establish the following relationships of harmonic powers (mean perihelion, mean, and mean aphelion* being designated by the subscript figures $1,2,3$ respectively).

Stockwell's logarithmic values are:-

$$
\begin{array}{cccccc}
\text { Neptune } \ldots & \Psi_{3} & 1 \cdot 481951 \alpha & y_{1} & 1 \cdot 473327 \alpha^{\prime} \\
\text { Uranus } \ldots & \delta_{1} & 1 \cdot 262996 \beta & \delta_{3} & 1 \cdot 301989 \beta^{\prime} \\
\text { Saturn } & \ldots & h_{1} & \cdot 957973 \gamma & h_{2} & .979496 \gamma^{\prime} \\
\text { Jupiter } & \ldots & \psi_{3} & \cdot 734588 \delta & \psi_{2} & \cdot 716237 \delta^{\prime} \\
\frac{1}{3}(\alpha-\beta)+\frac{1}{2}\left(\beta^{\prime}-\gamma\right)+\left(\delta-\gamma^{\prime}\right)=\cdot 000085=\log 1 \cdot 0002 \\
\frac{1}{3}\left(\alpha^{\prime}-\beta\right)+\frac{1}{2}\left(\beta^{\prime}-\gamma\right)+\left(\delta^{\prime}-\gamma\right)=\cdot 000382=\log 1 \cdot 0009
\end{array}
$$

These results represent the following equations of distance :-

$$
\left(\frac{\Psi_{3}}{\hat{\sigma}_{1}}\right)^{\frac{1}{3}} \times\left(\frac{\hat{\vartheta}_{3}}{\zeta_{1}}\right)^{\frac{1}{2}} \times\left(\frac{\Psi_{3}}{h_{2}}\right)^{\frac{1}{1}}=1 ; \quad\left(\frac{\Psi_{2}}{\hat{\sigma}_{1}}\right)^{\frac{3}{3}} \times\left(\frac{\Psi_{3}}{h_{1}}\right)^{\frac{1}{2}} \times\left(\frac{\psi_{2}}{h_{1}}\right)^{\frac{1}{1}}=1 .
$$

The theoretical differ from the actual values by less than $\frac{l}{50}$ of 1 per cent. in the first, and by less than $\frac{1}{11}$ of 1 per cent. in the second equation. The closeness of these agreements may, perhaps, induce a glance at a few that are somewhat more intricate.

If we substitute for the theoretical primitive figurate exponents $(1,3,6,10)$ the present actual vector radii $\left(a=\Psi_{2}\right.$; $\left.b={ }_{2} ; c=h_{2} ; d=4_{2}\right)$, we may form an equation for Saturn's mean perihelion :-

$$
\Psi_{2}^{d} \times{ }_{2}^{b-d} \times \psi_{2}=h_{1}^{a+b} \text {. }
$$

If $a, b, d$ represent the mean aphelion vector radii, the equation represents Saturn's mean distance :-

$$
\Psi_{3}^{d} \times \dot{\theta}_{3}^{b-d} \times \psi_{3}^{a}=h_{1}^{a+b} \text {. . . . . }
$$

If we take powers of the masses instead of powers of the vector radii, equation (2) yields two values for Saturn's mass, according as we use Newcomb's greatest or least value of Neptune's mass -

the greatest $\left(\frac{1}{19} \frac{1}{8} 0\right)$, deduced from $\psi$ 's satellite, . . (3) or the least $\left(\frac{1}{10} \overline{7}_{0}\right), \quad, \quad, \quad$ perturbations of ${ }^{\circ}$. (4) These four equations give approximations to precise accu* Snithsonian Contributions, 232, p. 38. 
racy, varying between $\frac{3}{5}$ and $\frac{1}{20}$ of 1 per cent., as will be seen by the following solutions :-

(1)

$$
\begin{aligned}
& \log 30 \cdot 03386^{5 \cdot 202798} \quad \text {. . . . . . . . } \mathbf{7} 687712
\end{aligned}
$$

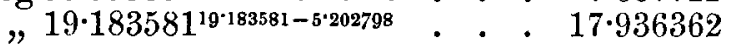

$$
\begin{aligned}
& \text { " } 5 \cdot 202798^{30 \cdot 03386} \text {. . . . . . . } 21.511361 \\
& 47 \cdot 135435 \\
& \text {, } 9 \cdot 077645^{30 \cdot 03386+19^{\cdot 183581}} \text {. . . } 47 \cdot 148979 \\
& (47 \cdot 148979-47 \cdot 135435) \div(30 \cdot 03386+19 \cdot 183581) \\
& =\log 1 \cdot 00051 \text {. }
\end{aligned}
$$

$$
\begin{aligned}
& \log 30 \cdot 33551^{5 \cdot 427351} \text {. . . . . . } 8 \cdot 043068
\end{aligned}
$$

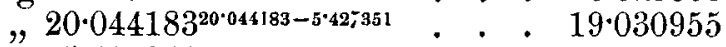

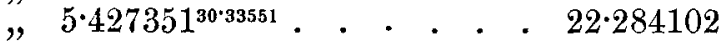

$$
\begin{aligned}
& 49 \cdot 358125 \\
& \text { " } 9 \cdot 538852^{30 \cdot 33551+20^{\circ} \cdot 041183} \text {. . . } 49 \cdot 346714 \\
& (49 \cdot 358125-49 \cdot 346714) \div(30 \cdot 33551+20 \cdot 044183) \\
& =\log 1 \cdot 0005 \text {. }
\end{aligned}
$$

\section{(3), (4)}

If $10=\log$ of sun's mass, the logarithms of the assumed mass are :-

$$
\begin{aligned}
& \text { ₹ (Nowcomb, from satellite) . . } 5 \cdot 712646 \\
& \Psi \text { ( }, \quad, \text { perturbations). 5.705534 } \\
& \text { ○ ( " ). . . . . . . } 5 \cdot 645892 \\
& \text { 々 (Bessel) . . . . . . . . 6.455734 } \\
& 4(,) \text {. . . . . . . . } 69979689
\end{aligned}
$$

Substituting these logarithms in equation (2), we get for logarithm of Saturn's mass, by using

Satellite value of $\log \Psi \quad$. (3) . $\quad 6.458198$

Perturbation value of $\log \Psi$. (4) . $\quad 6.456439$

$$
\begin{aligned}
& 6 \cdot 458198-6 \cdot 455734=\cdot 002464=\log 1 \cdot 0057 \\
& 6 \cdot 456439-6 \cdot 455734=\cdot 000705=\log 1 \cdot 0016 .
\end{aligned}
$$

These relations are suggestive of the many familiar equations between the sums of periodic times. The substitution of products for sums, and powers for products, in the distribution of mass and position, seems to indicate the early organizing activity of constant forces, acting with reference to nucleal centres, in elastic media.

Philadelphia, March 30, 1876. 